\title{
Efecto de la calidad de la información sobre la acreditación institucional: un estudio exploratorio en universidades chilenas
}

\author{
Effect of quality of information on institutional accreditation: \\ an exploratory study on Chilean universities
}

\author{
Emilio Rodríguez-Ponce $^{1} \quad$ Nicolas Fleet $^{2} \quad$ Liliana Pedraja-Rejas $^{3} \quad$ Juan Rodríguez-Ponce $^{4}$ \\ Recibido 30 de agosto de 2011, aceptado 25 de septiembre de 2012 \\ Received: August 30, 2011 Accepted: September 25, 2012
}

\begin{abstract}
RESUMEN
Este artículo discute acerca de la relación entre la calidad de la información y la calidad de las instituciones universitarias. Se propone que la gestión de la información en las instituciones universitarias es funcional y por lo tanto constituye un efecto notable sobre de su calidad global. Dicho efecto se describe de acuerdo a dos dimensiones, la primera: porque la información permite adoptar mejores decisiones para ajustar y mejorar los procesos institucionales; la segunda: porque la difusión de información sobre las universidades es estimulada cuando sus resultados se ajustan a parámetros de calidad reconocidos. Para comprobar estos planteamientos se relaciona la calidad de la información difundida con la calidad institucional global de las universidades chilenas, empleando una muestra de las 29 instituciones chilenas calificadas por el ranking Webometrics entre las 5000 mejores de acuerdo a la calidad de la información de sus páginas Web y considerando sus respectivos resultados de acreditación institucional. Se pretende probar que la información representa un efecto sobre la calidad de las universidades, el cual es empíricamente demostrable a través de los años de acreditación obtenidos. Como resultado se consigue un modelo explicativo donde efecto de la calidad de la información sobre la calidad institucional de las universidades alcanza un $62,8 \%$. Esto significa que existe una tendencia que vincula significativamente la calidad de la información con la calidad de las universidades.
\end{abstract}

Palabras clave: Calidad de la información, calidad institucional, Webometrics, gestión de la información, acreditación institucional.

\section{ABSTRACT}

This article discusses the relationship between quality of information and quality of university institutions. It is proposed that the management of information of university institutions is functional and therefore it constitutes a noteworthy effect on its overall quality. Such effect could be described according to two dimensions: firstly, because information enhances decision making in order to adjust and improve the institutional processes. Secondly, because the dissemination of information of universities is stimulated when their results meet recognized quality criteria. In order to test such statements the quality of information is correlated to the global institutional quality of Chilean Universities, using a sample of the 29 Chilean institutions ranked by Webometrics among the top 5000 in terms of the quality their Websites and considering the respective institutional accreditation results. As a result, an explanatory model is obtained, where the effect of quality of information on institutional quality of university, measured as years of accreditation, amounts to $62.8 \%$. This means that there is a trend significantly linking the quality of the information with the quality of the universities.

Keywords: Quality of information, institutional quality, Webometrics, management information, institutional accreditation.

1 Instituto de Alta Investigación. Universidad de Tarapacá. Arica, Chile. E-mail: erodrigu@uta.cl

2 Universidad de Cambridge. Cambridge, Reino Unido. Master of Science in Sociology. London School of Economics. University of London. E-mail: n.g.fleet@gmail.com

3 Escuela Universitaria de Ingeniería Industrial, Informática y de Sistemas. Universidad de Tarapacá. Arica, Chile. E-mail: lpedraja@uta.cl

4 Centro de Formación Técnica Tarapacá. Universidad de Tarapacá. Arica, Chile. E-mail: jrodrigup@uta.cl 


\section{INTRODUCCIÓN}

En la sociedad de la información y del conocimiento, las oportunidades de desarrollo individual, organizacional y de las sociedades, en general, están lógicamente determinadas por la capacidad de gestión sobre el conocimiento e información. En efecto, nuestra sociedad se caracteriza por la existencia de niveles de información y conocimiento crecientes. A su vez, al interior de las organizaciones, las personas poseen una racionalidad limitada, razón por la cual la gestión del conocimiento, al impactar sobre la base cognitiva de los equipos directivos, puede ser un determinante estructural de las elecciones estratégicas, el desempeño y los niveles de calidad. Así, el éxito de las organizaciones radica en su capacidad de gestionar la información que ellas producen para generar valor estratégico [11-12].

Las universidades, que son precisamente las organizaciones encargadas de generar y transferir el conocimiento e innovación necesarios para el desarrollo de las personas y los países en la sociedad del conocimiento, pueden también sostener su propia eficacia organizacional sobre la capacidad de gestionar conocimiento e información [13-15].

En este contexto, el presente artículo discute acerca del papel que la información juega específicamente en la calidad organizacional de las universidades en Chile. Adicionalmente, se espera que la discusión aporte a develar una parte específica de la compleja y multidimensional relación entre calidad de la educación superior y rendición de cuentas.

Ciertamente, existen diferentes maneras de vincular ambos términos: la rendición de cuentas puede obedecer a una estrategia de las universidades con prestigio para difundir públicamente las credenciales que la hacen acreedora del reconocimiento social [2]. También, la rendición de cuentas puede devenir en una cualidad del sistema de educación superior, en la medida en que las instituciones proveen información a los potenciales usuarios introduciendo mayores niveles de transparencia en los intercambios [22]. En otra dimensión, los procesos de acreditación, como principal política pública desarrollada en Chile para el aseguramiento de la calidad de la educación superior, ha tenido el efecto de incentivar la producción de información sobre la educación terciaria, en particular información relevante desde el punto de vista de la calidad, la cual es utilizada para la toma decisiones a nivel de la gestión institucional y académica, y para las elecciones de los postulantes [16]. Adicionalmente, la gestión y difusión de la información por parte de las universidades constituye una variable explicativa de la calidad organizacional de la educación superior.

En este artículo abordaremos esta última dimensión de la relación entre rendición de cuentas y calidad de la educación y, por tanto, se espera contribuir con evidencia empírica a partir del caso chileno mostrando exploratoriamente el efecto de la calidad de la información generada y difundida por las universidades, de acuerdo al ranking Webometrics, y su calidad institucional lograda, medida por los años de acreditación alcanzados en la evaluación realizada por la Comisión Nacional de Acreditación de Chile [4].

\section{LA CALIDAD DE LA INFORMACIÓN Y LA CALIDAD DE LAS INSTITUCIONES}

Existen discusiones sobre educación superior que son fundamentales porque buscan resolver preguntas sobre su valor intrínseco para la reproducción e innovación cultural de la sociedad. Por ejemplo, ¿cuál es el valor agregado de la educación superior? Para responder esta pregunta, las investigaciones reúnen evidencia empírica para comparar el efecto de los factores internos como lo es la propia educación impartida versus los factores externos tales como capital cultural, origen familiar y condición socioeconómica, y el respectivo impacto de estos factores sobre la inserción y desempeño laboral de los egresados [5]. Otro ejemplo, ¿qué dimensiones representan el logro o resultados de la educación superior? Esta discusión ha estado a la base de la confección de rankings de instituciones de educación superior en el mundo, los cuales al mismo tiempo que son ampliamente criticados [9] son universalmente utilizados.

La pregunta que queremos abordar en este apartado es sobre los factores que explican la calidad de la educación superior. Desde luego, se trata de un espacio vasto de discusión e investigación del que no podemos hacernos íntegramente cargo. El concepto mismo de calidad resulta en extremo difícil de definir y operacionalizar. En efecto, el reciente 
desarrollo de los procesos de acreditación en Chile, así como en otros países del mundo, se ha asociado a debates acerca del foco de evaluación [17]. La acreditación se basa en un concepto multidimensional de calidad institucional [7]. En particular, la evaluación de la acreditación institucional considera a las instituciones de educación superior como unidad organizacional, de modo que la calidad se verifica en la articulación concreta de sus procesos y resultados, sin necesariamente generar una regla estándar. Esta característica ha motivado, a nivel mundial, presiones por estandarizar los criterios de evaluación en indicadores concretos de resultados, de manera de "parametrizar" la evaluación ¿Será posible así dar con los indicadores fundamentales de la calidad? Si fuera esto posible, sin duda las instituciones, orientadas a fortalecer su reputación en el contexto de mercados competitivos, se esforzarían por satisfacer estos indicadores de la mejor manera posible [20]. Un problema de reducir la calidad a medidas acotadas es sobrevalorar aspectos que no siempre están dentro del ámbito de gestión de las instituciones universitarias. Por ejemplo, los resultados de acreditación podrían correlacionarse más con la selectividad o los tipos de estudiantes que las instituciones reciben que con la efectividad de la docencia impartida. En consecuencia, la pregunta por la calidad evaluada en los procesos de acreditación debe centrarse en los aspectos "internos", es decir, aquellos que dependen de la gestión de las universidades. Proponemos que la calidad de la información constituye una de estas dimensiones subyacentes de la calidad de la educación superior que es atribuible a la gestión directa de las instituciones universitarias.

La gestión de la información se articula en dos fases: la generación de sistemas de información y la difusión de la información. Como primera fase, la emergencia de sistemas de información con frecuencia ha obedecido a la necesidad que la participación en los procesos de acreditación institucional instala de generar evidencia que permita hacer seguimiento de la gestión y de los procesos académicos. La acreditación estimula la generación de información sobre la calidad, esto es, que las instituciones de educación superior recopilen, organicen y articulen de manera significativa datos e indicadores representativos de los resultados obtenidos por sus mecanismos de aseguramiento de la calidad [16]. Por lo tanto, la disponibilidad de un sistema de información es un indicador de la capacidad institucional de introducir mejoramientos en distintos niveles de su gestión. Más aún, la evidencia internacional muestra que los procesos de acreditación impactan sobre el mejoramiento de la calidad de gestión de la información [19].

Ciertamente que la etapa de generación de sistemas de información es finalmente relevante para la calidad de las universidades en la medida en que apoya el proceso de toma de decisiones. Como se plantea en $[10,16]$, la eficacia del equipo de alta dirección de una universidad depende de la capacidad para crear, compartir y aplicar conocimientos, siendo esta capacidad un potencial determinante estructural de las elecciones estratégicas, el desempeño y los niveles de calidad que alcance la organización universitaria en su conjunto.

Por su parte, la fase de difusión de información constituye una premisa fundamental para el funcionamiento de cualquier mercado y especialmente en el caso de la educación superior. Ello, de acuerdo a [6], porque la educación superior provee "bienes de experiencia", tales como los programas académicos, de modo que la información es crucial para que los estudiantes puedan tomar decisiones de una mejor manera. Así, en [1] se sostiene que un sistema de educación superior altamente diversificado, con instituciones que varían considerablemente en el tipo de oferta educativa, prestigio y calidad y cuyo principal medio regulador es la competencia, constituye un sistema exigente en cuanto a demandas de información.

A nivel de la difusión, es necesario distinguir entre información y publicidad. Según [18], el fin de la publicidad no es ofrecer información objetiva acerca de la calidad de una institución en particular, sino que provocar una percepción favorable en los potenciales usuarios, con el fin de incrementar su prestigio en el marco de la competencia por postulantes, académicos, fondos, etc. En cambio, por información entendemos los datos e indicadores objetivos sobre procesos y resultados institucionales, cuya significación depende de información de carácter cualitativa sobre las definiciones y propósitos fundamentales que orientan el quehacer de las universidades. $\mathrm{La}$ difusión de información se distingue por aportar antecedentes y datos relevantes para la toma de decisiones de los usuarios. 
No es común encontrar en las páginas Web de las universidades del país un espacio especialmente dedicado a la provisión de información y datos institucionales. Una primera explicación de este efecto radica en la falta de información de las universidades sobre sus propios procesos y resultados, es decir que no hayan completado la primera fase de la gestión de la información. Una segunda explicación es que las universidades, aun disponiendo de los datos, optan por no informar, por la aprensión de exhibir debilidades. De esta manera, las universidades pueden privilegiar difundir determinada información o bien publicidad que les permita proyectar una "apariencia" ideal acorde a un patrón predominante. En educación superior este patrón normativo genérico suele obtenerse de las universidades fuertes en excelencia e investigación, que lideran los ranking a nivel nacional e internacional y cuya tradición es capitalizada en términos de prestigio institucional, generando un efecto de reducción de la potencial diversidad de las universidades en un grupo específico de medidas de éxito, prestigio y calidad [23]. Con ello, se inhibe la difusión de información sobre, por ejemplo, el segmento no selectivo (masivo o no de elite) [21] de la educación superior y sus potenciales resultados en términos de la eficacia u orientación específica del proceso de formación.

Entonces, en la medida que la difusión de la información se asocia a un concepto predominante de calidad, basado en excelencia académica e investigación, posiblemente otras variantes de la calidad institucional son menos difundidas. Ello ciertamente plantearía una dificultad al intento de establecer la relación entre información y calidad institucional de una manera unívoca. Es decir, el efecto de la gestión y difusión de la información sobre la calidad institución estaría sobredeterminado por la tendencia consistente en que las universidades con altos niveles de calidad, y por lo tanto, desempeños satisfactorios en indicadores socialmente relevantes (tales como: investigación, selectividad y calidad académica) informan públicamente acerca de estos resultados.

El foco de nuestro análisis está puesto en la fase de difusión de la información, la cual contiene la anterior fase de generación de sistemas de información. Es decir, el indicador más claro de la efectividad de la gestión de la información de una universidad está en la difusión que ésta hace de sus principales procesos y resultados. Nuestra hipótesis es que el nivel de la gestión de información de una universidad puede medirse a partir del nivel de difusión de información y siendo esta información publicada en función del interés de las instituciones de dar cuenta de su calidad (cuando ella es efectivamente lograda), la difusión de información es una variable que explica la calidad de la educación superior. Así, pretendemos demostrar que es posible acceder a una dimensión subyacente de la calidad institucional. En corto, entre calidad y rendición de cuentas hay una relación de interdependencia, la cual es empíricamente demostrable.

\section{METODOLOGÍA DEL ESTUDIO}

\section{Muestra}

Se trabaja con las 29 universidades chilenas calificadas, por el ranking Webometrics (www. webometrics.info/), entre las 5.000 mejores instituciones en la calidad de sus páginas Web. Este es un ranking que considera el tamaño, la visibilidad, la riqueza de la información general y la calidad de la información académica de cada una de las universidades del mundo.

\section{Variables}

La calidad de la información se mide rankeando, entre sí, las universidades chilenas siguiendo el orden del ranking Webometrics. La calidad de las instituciones considera los años de acreditación institucional vigentes otorgados por la Comisión Nacional de Acreditación de Chile. A su turno, los años de acreditación institucional (de 1 a 7) expresan de manera directamente proporcional la evaluación cualitativa de los procesos y resultados obtenidos por las instituciones de educación superior, que se realiza como mínimo para los ámbitos de gestión institucional (que incluye la disponibilidad de información para la toma de decisiones) y de docencia de pregrado (que incluye la disponibilidad de información sobre efectividad del proceso de formación y seguimiento de egresados).

Así, la información empleada fue la siguiente: 
Tabla 1. Universidades chilenas calificadas por el ranking Webometrics.

\begin{tabular}{|c|c|c|}
\hline Institución & $\begin{array}{c}\text { Lugar en } \\
\text { el Ranking } \\
\text { Nacional } \\
\end{array}$ & $\begin{array}{c}\text { Años de } \\
\text { Acreditación }\end{array}$ \\
\hline Universidad de Chile & 1 & 7 \\
\hline Universidad de Concepción & 2 & 6 \\
\hline $\begin{array}{l}\text { Pontificia Universidad } \\
\text { Católica de Chile }\end{array}$ & 3 & 7 \\
\hline $\begin{array}{l}\text { Universidad Austral de } \\
\text { Chile }\end{array}$ & 4 & 6 \\
\hline $\begin{array}{l}\text { Universidad Técnica } \\
\text { Federico Santa María }\end{array}$ & 5 & 6 \\
\hline $\begin{array}{l}\text { Universidad de } \\
\text { La Frontera }\end{array}$ & 6 & 5 \\
\hline $\begin{array}{l}\text { Pontificia Universidad } \\
\text { Católica de Valparaíso }\end{array}$ & 7 & 6 \\
\hline $\begin{array}{l}\text { Universidad de Santiago } \\
\text { de Chile }\end{array}$ & 8 & 6 \\
\hline Universidad de Talca & 9 & 5 \\
\hline Universidad del Bío Bío & 10 & 5 \\
\hline $\begin{array}{l}\text { Universidad Alberto } \\
\text { Hurtado }\end{array}$ & 11 & 5 \\
\hline Universidad de Valparaíso & 12 & 4 \\
\hline Universidad Diego Portales & 13 & 5 \\
\hline Universidad Andrés Bello & 14 & 5 \\
\hline Universidad de Tarapacá & 15 & 5 \\
\hline Universidad de Antofagasta & 16 & 4 \\
\hline $\begin{array}{l}\text { Universidad Católica } \\
\text { del Norte }\end{array}$ & 17 & 6 \\
\hline Universidad Arturo Prat & 18 & 2 \\
\hline $\begin{array}{l}\text { Universidad Central } \\
\text { de Chile }\end{array}$ & 19 & 4 \\
\hline $\begin{array}{l}\text { Universidad Católica } \\
\text { del Maule }\end{array}$ & 20 & 5 \\
\hline $\begin{array}{l}\text { Universidad Metropolitana } \\
\text { de Ciencias de la } \\
\text { Educación }\end{array}$ & 21 & 4 \\
\hline $\begin{array}{l}\text { Universidad Católica } \\
\text { de Temuco }\end{array}$ & 22 & 5 \\
\hline Universidad del Desarrollo & 23 & 5 \\
\hline Universidad de Los Lagos & 24 & 3 \\
\hline $\begin{array}{l}\text { Universidad de Playa } \\
\text { Ancha }\end{array}$ & 25 & 3 \\
\hline $\begin{array}{l}\text { Universidad Tecnológica } \\
\text { de Chile }\end{array}$ & 26 & 3 \\
\hline $\begin{array}{l}\text { Universidad de Viña } \\
\text { del Mar }\end{array}$ & 27 & 3 \\
\hline Universidad de Atacama & 28 & 2 \\
\hline $\begin{array}{l}\text { Universidad Católica de la } \\
\text { Santísima Concepción }\end{array}$ & 29 & 4 \\
\hline
\end{tabular}

Fuente: www.webometrics.info/

\section{Métodos}

Empleando la Tabla 1, basada en fuentes de información secundaria, se aplica una ecuación de regresión para verificar la capacidad explicativa que tiene la calidad de la información sobre la calidad institucional. La ecuación utilizada fue:

\section{Calidad de la Institución $=\mathrm{A}+\mathrm{b}_{1} *$ Lugar en el ranking de calidad de la información $+\mathrm{E}_{\mathrm{b}}$}

El coeficiente $A$ comprende las variables omitidas por el modelo; $b_{1}$ es el factor de ponderación de la variable independiente y $E_{i}$ es el error residual del modelo, el cual representa la parte del error de estimación que no es capturado por el modelo de regresión simple.

\section{PRINCIPALES RESULTADOS}

Los resultados de la aplicación de la ecuación de regresión son los siguientes:

Calidad institucional $=6,559-0,125 *$ Lugar en el Ranking Nacional Webometrics

Estos resultados implican que cuanto más arriba se esté en el ranking nacional Webometrics, mayores serán los años de acreditación institucional. Es decir, la calidad de la información representa un efecto sobre la calidad institucional, ya que un mejor lugar en el ranking Webometrics se relaciona con mayores años de acreditación.

Esta ecuación de regresión es significativa (Test $\mathrm{F}=45,587 ; \mathrm{P}<0,001)$, toda vez que el modelo explica el 62,8\% de las variaciones en la variable dependiente (número de años de acreditación). Existen variables omitidas en el modelo, pero la calidad de la información, representada por el lugar en el ranking Webometrics, es una variable significativa para explicar la calidad institucional (Test $=-6,752 ; \mathrm{P}<0,001)$.

Estos resultados se sintetizan en la Tabla 2. 
Tabla 2. Análisis de regresión.

\begin{tabular}{|l|c|c|c|c|c|c|}
\hline & $\begin{array}{c}\text { Coeficiente no } \\
\text { estandarizado }\end{array}$ & Error típico & Test t & Significancia & R2 & Test F \\
\hline Cons-tante & 6,559 & 0,317 & 20,689 & 0,000 & & \\
\hline Ran king & $-0,125$ & 0,018 & $-6,572$ & 0,000 & & \\
\hline Modelo & & & & & 0,628 & 45,585 \\
\hline
\end{tabular}

\section{CONCLUSIONES}

Como conclusión central de este estudio se sostiene que la gestión de la información, como proceso que culmina en la difusión y uso de la información, es una variable relevante para caracterizar la calidad de las universidades.

Para probar esta idea, se presentaron los resultados de un modelo donde la calidad de la información, representada por el lugar de la institución en el ranking Webometrics, ingresa como la variable que explica la calidad de las universidades, representada por los resultados de acreditación institucional. La capacidad explicativa del modelo alcanzó el 62,8\%. Ello significa que la gestión de la información es una variable determinante de la calidad institucional en gran medida.

Se podría contraargumentar que la relación propuesta por el modelo de regresión entre difusión de información y calidad es espuria, en el sentido de que podría existir otra variable explicativa anterior a la gestión de la información que determine la verdadera razón de los resultados obtenidos. Pero justamente sostenemos que la difusión de la información es una variable relevante que agrupa y es explicativa de otras variables de la calidad. Desde luego, la difusión de la información permite explicar la existencia de sistemas de información. Si no hay difusión de información, frecuentemente es porque no hay capacidad de generarla. Pero también la difusión de la información ocurre cuando hay resultados adecuados a los estándares de calidad predominantes. $\mathrm{Si}$ es que no se percibe calidad, probablemente no exista una actitud espontánea (como es la que rige lo que se publica en las páginas Web) para difundir la información. En consecuencia, la correlación entre difusión de información y calidad obedece a que i) efectivamente la información constituye una dimensión relevante, y no suficientemente considerada, de la calidad institucional; y ii) que a mayor calidad, las instituciones buscan capitalizarla en términos de prestigio, difundiendo públicamente sus resultados. En este sentido, la información sirve como eslabón entre calidad, rendición de cuentas y prestigio. $\mathrm{O}$, más bien, la orientación hacia la rendición de cuentas coincide y se refuerza con la orientación hacia el aseguramiento de la calidad.

Como hemos planteado, el efecto de la difusión de la información sobre la calidad institucional podría estar sobredeterminado por un patrón predominante de calidad institucional, en el sentido de que las universidades de más prestigio acumulado en términos de excelencia académica, preferencia de los estudiantes de elite y resultados sostenidos en investigación suelen reflejar mayores niveles de calidad que aquellas universidades que, independientemente de contar con mecanismos de aseguramiento de la calidad, por el hecho de recibir a estudiantes menos preparados de la educación secundaria y concentrarse en ofrecer un tipo de docencia orientada a promover la movilidad social y la colocación laboral de los egresados, puedan aparece como comparativamente de menor calidad. En otras palabras, las universidades adecuadas al modelo predominante de calidad tienen, por esta razón, mayores incentivos a difundir información pública. Ello constituiría una variable que interviene en el modelo de regresión, la que puede ser proviosionalmente denominada como "orientación al prestigio" o "autopresentación institucional", y que sin embargo no es operacionalizada ni medida en su contribución específica al efecto general.

Sin embargo, la sobredeterminación que la orientación al prestigio pueda ejercer sobre la difusión de información no solo constituye un problema de medición ni se resuelve únicamente como tal, pues es también un problema de política de calidad universitaria, en el sentido de la necesidad de establecer y promover buenas prácticas de gestión de la información, que permitan asociar información cuantitativa que es generada a partir de los procesos y resultados instituciones con definiciones cualitativas 
que proporcionen significación y propósito. Ello tendría efectos positivos sobre el mejoramiento de la calidad de la educación superior y, al mismo tiempo, contribuirá a valorar una diversidad mayor de modelos y parámetros sobre lo que puede llegar a ser educación superior de calidad, tanto en el segmento de elite como masivo del sistema.

Un ejemplo de políticas que favorecen las prácticas de difusión, por lo tanto también de gestión de información, es la Ley 20.285 sobre transparencia y acceso a la información pública, que establece un conjunto de datos mínimos de gestión institucional que todas las instituciones públicas, incluyendo universidades públicas, deben poner a disposición libre de los usuarios en los respectivos sitios Web. De esta manera, de los 29 primeros lugares en el ranking nacional de Webometrics, 13 corresponden a universidades públicas, las que a su vez corresponden al $81 \%$ del total de universidades públicas del país (16). Las buenas prácticas de difusión de información tienen valor como tales y, como sugerimos en este artículo, pueden contribuir a la calidad institucional por la vía del mejoramiento de la toma de decisiones y la orientación al prestigio (es decir, la motivación a mejorar procesos y resultados por la exposición pública). Aun cuando existen un par de casos de resultados relativamente bajos en calidad ( 2 años de acreditación) con altos niveles de difusión de información, la mayoría de las universidades públicas exhiben también altos niveles de calidad en sus respectivos resultados de acreditación, reforzando así el efecto verificado con el modelo de regresión.

\section{AGRADECIMIENTOS}

Esta investigación es resultado del proyecto de investigación FONDECYT 1090116 financiado por la Comisión Nacional de Investigación Científica y Tecnológica (CONICYT, Chile). Asimismo los autores agradecen la colaboración de Milagros Delgado Almonte, Ing., MBA (Universidad de Chile), y Elisa Palma Sánchez, Psic., MA (University of Newcastle), por sus valiosos comentarios y sugerencias.

\section{REFERENCIAS}

J.J. Brünner. "Políticas y mercado de educación superior: necesidades de información". En J.J. Brünner y P. Meller (eds.). Oferta y demanda de profesionales y técnicos en Chile. El rol de la información pública. Santiago: Ril editores, pp. 19-85. 2004.

[2] J.J. Brünner. "Educación superior en Chile: Instituciones, mercados y políticas gubernamentales, 1967-2007". Universidad de Leiden, p. 387. Holanda. 2008.

[3] M. Castells. "The rise of the network society". Malden, MA: Blackwell Publishing, p. 594. 2004.

[4] Comisión Nacional de Acreditación. "Información en las páginas Web de las universidades autónomas chilenas". Documento de trabajo CNA-Chile, p. 39. 2010.

[5] D. Contreras. "Explaining wage inequality in Chile: does education really matter?". Journal of Development Studies. Vol. 11, Issue 1-2, pp. 53-76. 2002.

[6] D. Dill and M. Soo. "Transparency and quality in Higher Education markets". Higher Education Dynamics, Douro Series. Netherlands, pp. 61-86. 2004.

[7] L. Harvey and D. Green. "Defining Quality. Assessment \& evaluation in Higher Education". Vol. 1, pp. 9-26. 1993.

[8] IPSOS-Chile. "Estudio exploratorio sobre efectos de la acreditación institucional en la calidad de la educación superior en Chile". Documento de trabajo CNA-Chile, p. 161. 2010.

[9] S. Marginson and M. Van Der Wende. "To Rank Or To Be Ranked: The Impact of Global Rankings. Higher Education". Journal of Studies in International Education. Vol. 11, Issue 3-4, pp. 306-329. 2007.

[10] L. Pedraja-Rejas and E. Rodríguez-Ponce. "Leadership styles, knowledge management and strategy design: an empirical study in small and medium-sized firms". Interciencia. Vol. 33, Issue 9, pp. 651-657. 2008.

[11] L. Pedraja-Rejas, E. Rodríguez-Ponce and J. Rodríguez-Ponce. "The influence of knowledge management on the organizational performance: A study in public institutions and private firms". Revista Facultad de Ingeniería-Universidad de Antioquia. Vol. 47, pp. 218-227. 2009 a.

[12] L. Pedraja-Rejas, E. Rodríguez-Ponce and J. Rodríguez-Ponce. "Knowledge management and organizational efficacy in small and 
medium enterprises". Revista Venezolana de Gerencia. Vol. 14 No 48, pp. 495-506. 2009 b.

[13] E. Rodríguez-Ponce. "Knowledge management and organizational performance: An empirical study in public institutions". Interciencia. Vol. $32 \mathrm{~N}^{\circ} 12$, pp. 820-826. 2007.

[14] E. Rodríguez-Ponce. "The role of universities in the knowledge society and globalization: Evidence from Chile". Interciencia. Vol. 34 No $^{\circ}$, pp. 822-829. 2009.

[15] E. Rodríguez-Ponce. "Las universidades en la sociedad del conocimiento". A. Arata y E. Rodríguez-Ponce (eds.). Desafíos y perspectivas de la dirección estratégica de las instituciones universitarias. Santiago: Ediciones CNA-Chile, pp. 35-68. 2009.

[16] E. Rodríguez-Ponce, N. Fleet y M. Delgado. "La acreditación en la generación de información sobre la calidad de la educación superior". Revista Calidad de la Educación". No 31, pp. 212-230. 2009.

[17] E. Rodríguez-Ponce, N. Fleet y M. Delgado. "Capacidad predictiva de la evaluación de los pares y focos del modelo de acreditación institucional en Chile" Revista da Avaliação da Educação Superior. Vol. 15 N $^{\circ}$ 1, pp. 121141. 2010.
[18] A. Squella. "La información en el marco de la calidad de la educación superior. Información en la educación superior: necesidades y propuestas". Consejo Superior de Educación, serie Seminarios Internacionales. $N^{\circ} 5$, pp. 22-32. 2001.

[19] B. Stensaker. "Outcomes of quality assurance: a discussion of knowledge, methodology and validity". Quality in Higher Education. Vol. 14, Issue 1, pp. 3-13. 2008.

[20] M. Trow. "Trust, markets, and accountability in Higher Education: a comparative perspective". Higher Education Policy. Vol. 9, Issue 4, pp. 309-324. 1996.

[21] M. Trow. "Elite to mass to universal access: forms and phases of higher education in modern societies since WWII". In J. Forest and P. Altbach (eds.) International Handbook of Higher Education, pp. 243-280. 2011.

[22] G. Zapata. "Información en educación superior". Revista Calidad de la Educación. $\mathrm{N}^{\circ}$ 24, pp. 199-213. 2006.

[23] G. Zapata y N. Fleet. "Mercado, accountability e información pública en educación superior". Ponencia presentada en el Primer Congreso Chileno de Investigación en Educación Superior, p. 20. 20 de octubre de 2009. 